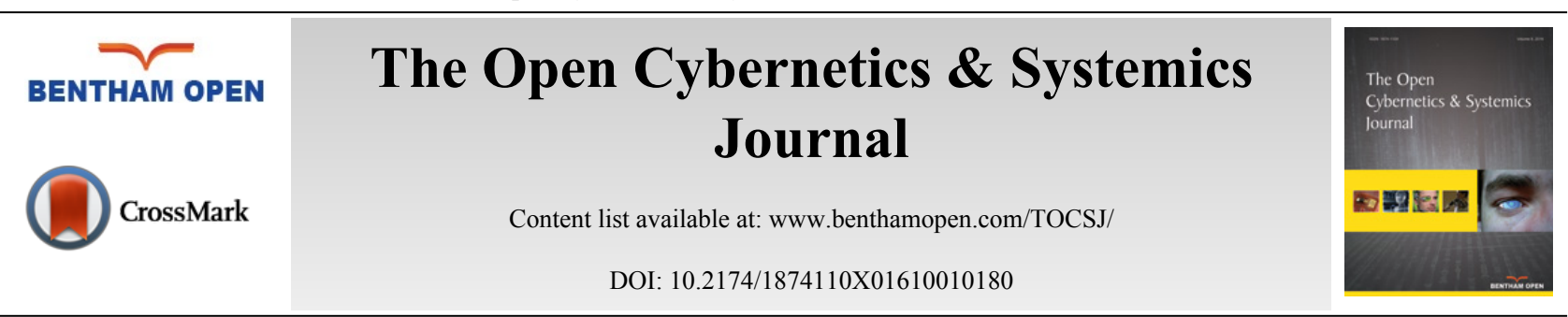

RESEARCH ARTICLE

\title{
A Parallel Hybrid Controller based on the Backstepping Method and S-plane Control for Three-dimensional Tracking of a Semi-pelagic Trawl System
}

\author{
Zhao Yan ${ }^{*}, 1$, Yunsheng $\mathrm{Mao}^{2}$ and Shaohong $\mathrm{Li}^{2}$ \\ ${ }^{1}$ Key Laboratory of High Performance Ship Technology, Wuhan University of Technology, Ministry of Education, \\ Wuhan, Hubei Province, 430063, China \\ ${ }^{2}$ Wuhan University of Technology, Wuhan, Hubei Province, 430063, China
}

Received: March 18, 2016

Revised: July 9, 2016

Accepted: August 21, 2016

\begin{abstract}
Aiming at improving the security of a semi-pelagic trawl in a complex working environment, this paper proposes a threedimensional tracking control system for the guidance of a semi-pelagic trawl on a complicated trajectory. The flexible nonlinear trawl system is simplified as a mass-spring-bar model. A hierarchical backstepping controller is then designed for the trajectory tracking of the trawl net and two otter boards. However, high-order state variables caused by the non-strict feedback characteristic of the trawl system appear frequently in recursive processes. Hence, a control algorithm based on the Sigmoid function is applied to construct the control outputs of these high-order variables. The stability of the proposed hybrid control method is analyzed based on the Lyapunov theorem when interference with an unknown upper limit occurs. Finally, simulation and contrast examinations show that the control algorithm is effective.
\end{abstract}

Keywords: Backstepping method, Lyapunov function, S(Sigmoid)plane control, Semi-pelagic trawl, Sigmoid function, Threedimensional tracking.

\section{INTRODUCTION}

The semi-pelagic trawl is a type of relatively advanced trawl system. It is mainly adopted to catch demersal fish such as squid and butterfish. Compared with the bottom trawl, it has proved to be friendly to the undersea environment [1]. Similar to the mid-water trawl, the semi-pelagic trawl system is hydrodynamically balanced, which means that the sweeps, bridles and trawl net maintain a short distance from the seabed, but the otter boards are still on the sea bottom [2]. The diagrammatic sketch of a semi-pelagic trawl system is shown in Fig. (1). In the process of trawling, rough undersea mountains and some obstacles such as subsea production equipment and pipelines sometimes can hook the otter boards, which will affect the catch rate and damage the trawl net. This situation may even threaten the safety of the vessel in bad weather. The purpose of this work is to study a three-dimensional trajectory tracking approach for a semipelagic trawl system, which can control the trajectories of the otter boards and trawl net. Owing to the complexity of the semi-pelagic trawl system, it is difficult for a single control strategy to achieve this control objectives, so a combined control method is needed. The otter boards can then leave the seabed and the trawl net can follow a complicated trajectory. After a collision with the seabed, the trawl system can rapidly return to a steady state of motion.

Tracking control of the trawl system is difficult because of the hulking physical size of the entire system and the flexibility of the trawl net and warps. At present, a few approaches have been studied. In the study of control modeling, Umeda proposed a first-order simplified model to control the depth of the trawl net [3]. Lee et al. presented a nonlinear

\footnotetext{
* Address correspondence to this author at the Key Laboratory of High Performance Ship Technology, Wuhan University of Technology, Ministry of Education, Wuhan, Hubei Province, 430063, China; Tel: 15827533198; E-mail: yanzhao19890907@163.com
} 
trawl mathematic model, which considers the trawler, otter board and net as three material points, and all ropes are simplified as elastic links $[4,5]$. This nonlinear model has been employed widely. In the study of control methods, several conventional PID controllers have been adopted to control the trajectory of the trawl net by regulating the otter board and considering the horizontal movement [6 - 8]. The fuzzy control approach and robust $\mathrm{H} 2 / \mathrm{H} \infty$ method have been attempted to realize controlled directed fishing with the mid-water trawl and semi-pelagic trawl [9, 10].

However, the above literature is mainly aimed at the depth control of the trawl net. To ensure the safety of the semipelagic trawl in a complex environment, trajectory tracking control in three-dimensional space is required. Moreover, the motion state parameters of two otter boards should be seriously considered because they provide the lateral spread of the trawl net. Few studies have investigated three-dimensional tracking of the semi-pelagic trawl. Nevertheless, many approaches have been proposed to control the trajectory of under-actuated unmanned underwater vehicles(UUV), which are quite similar to the research question in this work. The commonly used tracking control methods of UUVs are neural network adaptive control, sliding-mode control and the backstepping method. Yu et al. presented a direct adaptive control algorithm for UUVs based on a generalized dynamic fuzzy neural network [11]. Jia et al. approached a virtual guide method to establish the space motion error equation of UUVs, and a three-dimensional path controller was designed based on nonlinear iterative sliding mode [12]. Do et al. adopted the Lyapunov direct method and backstepping technique to design an adaptive path tracking controller of UUVs, which considered the parameter uncertainty of the system model [13]. Jon et al. combined the backstepping method and feedback control model to control slender-body under-actuated UUVs and carried out an experiment [14]. Zhu et al. proposed a backstepping tracking control algorithm for UUVs combined with a bio-inspired neurodynamics model, which reduced the speed jump in the conventional backstepping controller $[15,16]$. Xu et al. applied a virtual velocity to replace attitude tracking in the backstepping design, and adaptive sliding model control was adopted to increase the adaptive ability of UUVs in dynamic uncertain environments $[17,18]$.

In this work, the three-dimensional trajectory tracking strategy of a trawl net and otter boards are considered. First, a simple kinetic model of the semi-pelagic trawl system considering the motions in three degrees of two otter boards is established.

A hybrid control algorithm based on the hierarchical backstepping method and another nonlinear control method is then proposed. The backstepping method is used to design the virtual control variables of each subsystem from lower to higher orders. Owing to the non-strict feedback characteristics of the model state equation, the backstepping method is unavailable for some subsystems that contain high-order variables. A parallel nonlinear controller called "S-plane control" can solve these high-order variables directly.

Subsequently, the stability of the proposed control algorithm has been proved based on Lyapunov theory. To illustrate the superiority of the proposed control approach, a simulation experiment comparison is conducted based on the MATLAB/Simulink platform.

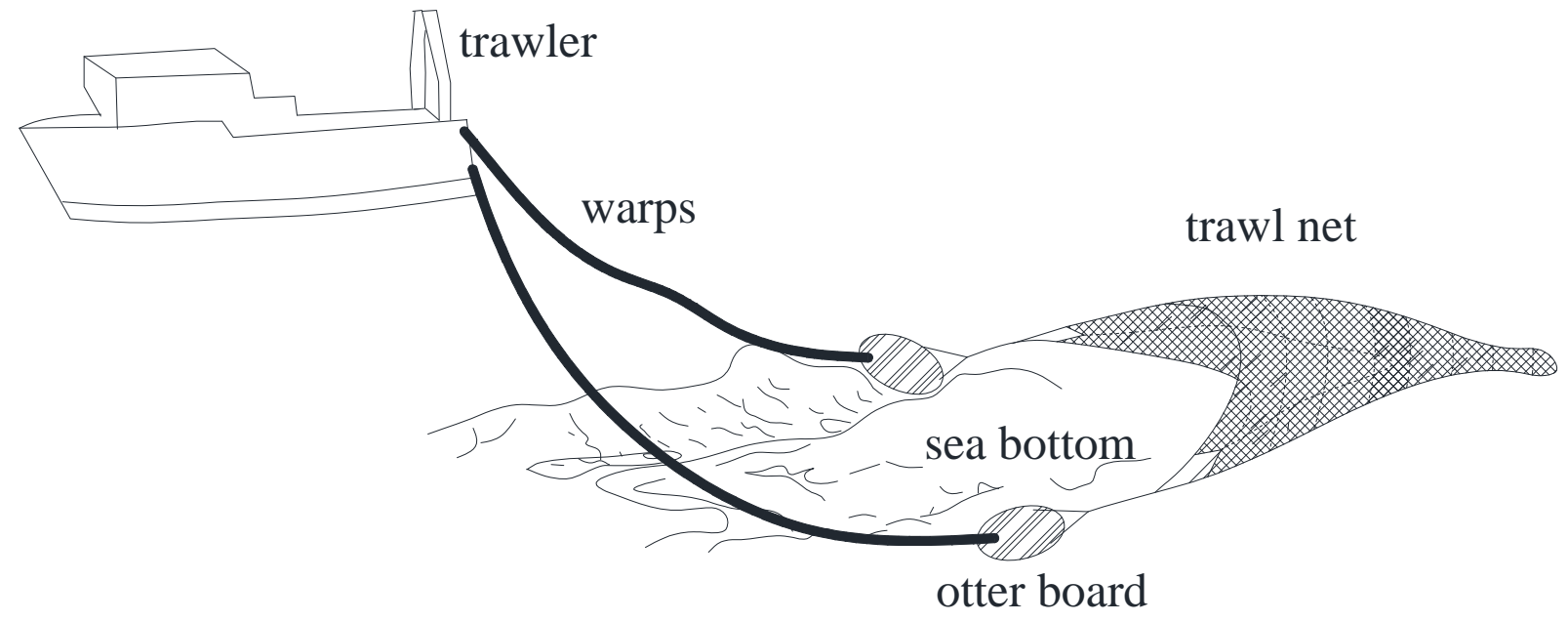

Fig. (1). Diagrammatic sketch of a semi-pelagic trawl. 


\section{MATHEMATICAL MODEL OF A SEMI-PELAGIC TRAWL}

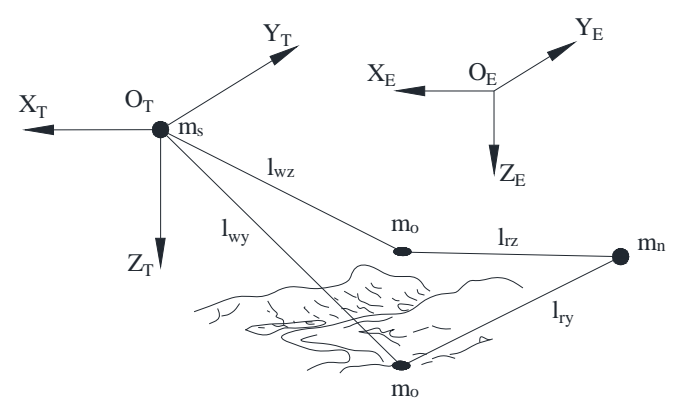

Fig. (2). Simplified model of a semi-pelagic trawl.

The semi-pelagic trawl is a complex, flexible and under-actuated system. A kinematic model is established based on the following simplified conditions. The trawl net and otter boards have planar motions in three degrees of freedom. The trawler moves in the horizontal plane with two translational degrees of freedom and one rotational degree of freedom. As shown in Fig. (2), two rectangular coordinate systems have been defined i.e., the earth fixed frame ( $X_{E}, Y_{E}$, $\left.Z_{E}\right)$ and the ship fixed $\left(X_{T}, Y_{T}, Z_{T}\right)$ frame. The trawler, two otter boards and trawl net are simplified as four mass points. The two pairs of warp and sweep are simplified as flexible links. The tension of all ropes can be calculated by Hooke's law. The simplified model of the semi-pelagic trawl can be named as the "four-mass-point model".

The state variables of the trawl system can be defined as:

$$
x=\left[\begin{array}{cccccc}
q_{n} & \dot{q}_{n} & q_{o z} & \dot{q}_{o z} & q_{o y} & \dot{q}_{o y} \\
q_{s} & \dot{q}_{s} & l_{w z} & i_{w z} & l_{w y} & i_{w y}
\end{array}\right]
$$

where $q_{n}$ and $\dot{q}_{n}$ are the position and velocity of the trawl net, $q_{o z}$ and $\dot{q}_{o z}$ are the position and velocity of the port otter boards, $q_{o y}$ and $\dot{q}_{o y}$ are the position and velocity of the starboard otter boards, and $q_{s}$ and $\dot{q}_{s}$ are the position and velocity of the trawler, respectively. $l_{w z}$ and $l_{w y}$ are the lengths of the two warps, and $i_{w z}$ and $i_{w z}$ are the release rates of these warps.

The external environment of the semi-pelagic trawl is rather complicated. The trawler is under the joint action of wind, waves and current. Based on the nonlinear mathematical model for ships [19], the kinetic equations of the trawler can be obtained.

The main source of the environmental disturbance of the otter boards and trawl net is the current. Based on D'Alembert's principle, the kinetic equations of the port otter board can be expressed as:

$$
m_{o} \ddot{q}_{o z}+B_{o}\left|\dot{q}_{o z}\right| \dot{q}_{o z}+W_{o}=k_{2}^{s z}\left(q_{s}-q_{o z}-l_{w z}\right)-k_{1}^{s}\left(q_{o z}-q_{n}-l_{r z}\right)+\omega_{o z}
$$

where $k_{2}^{s z}$ is the elasticity coefficient of the port sweep. $m_{0}, W_{0}$ and $B_{0}$ are the equivalent mass, the gravity and the resistance coefficient of the otter board, respectively. $\omega_{o z}$ is the environmental disturbance. The equations of the two otter boards are basically the same. When the system motion state changes, the fluid resistance coefficient $B_{0}$ is slowly varying. Owing to the limitation of fluid theory, $B_{0}$ is obtained by parameter estimation.

The kinetic equations of the trawl net mass point are similar to Eq. (2). According to the mechanical properties of the hydraulic winch, the kinetic equations of the port trawl winch can be expressed as:

$$
J_{w} \ddot{l}_{w z}=W_{d}-B_{w} \dot{l}_{w z}-k_{2}^{s z}\left(q_{s}-q_{o z}-l_{w z}\right) \cdot r_{w}
$$

where $J_{w}$ is the rotational inertia of the winch, and $W_{d}$ is the tension produced by the power source of the winch. $B_{w}$ is the damping coefficient, and $r_{w}$ is the radius of the roller.

Combined with the equations of these subsystems, the state-space equations of the three mass point models are 
obtained [20]. For the proposed four mass point models, the trawl net is pulled by two sweep lines. Analogously, the trawler is pulled by two warps. Hence the state equations of these two subsystems must be modified. The otter boards and the trawl winches both require two sets of equations to describe their motion states.

After the above modification, the state equations of the semi-pelagic trawl system can be expressed as:

$$
\left\{\begin{array}{l}
\dot{x}_{1}=x_{2} \\
\dot{x}_{2}=\left[-\hat{B}_{n} \cdot x_{2}^{2}-W_{n}+k_{1}^{s}\left(x_{3}-x_{1}-l_{r z}\right)+k_{1}^{s}\left(x_{5}-x_{1}-l_{r y}\right)+\omega_{n}\right] / m_{n} \\
\dot{x}_{3}=x_{4} \\
\dot{x}_{4}=\left[-\hat{B}_{o} \cdot x_{4}^{2}-W_{o}-k_{1}^{s}\left(x_{3}-x_{1}-l_{r z}\right)+k_{2}^{s z}\left(x_{7}-x_{3}-x_{9}\right)+\omega_{O z}\right] / m_{o} \\
\dot{x}_{5}=x_{6} \\
\dot{x}_{6}=\left[-\hat{B}_{o} \cdot x_{6}^{2}-W_{o}-k_{1}^{s}\left(x_{5}-x_{1}-l_{r y}\right)+k_{2}^{s y}\left(x_{7}-x_{5}-x_{11}\right)+\omega_{o y}\right] / m_{o} \\
\dot{x}_{7}=x_{8} \\
\dot{x}_{8}=\left[u_{s}-B_{s} \cdot x_{8}-C_{s} \cdot x_{8}+k_{2}^{s z}\left(x_{7}-x_{3}-l_{9}\right)+k_{2}^{s y}\left(x_{7}-x_{5}-x_{11}\right)\right] / M_{s} \\
\dot{x}_{9}=x_{10} \\
\dot{x}_{10}=\left[u_{w z}-B_{w} \cdot x_{10}-k_{2}^{s z}\left(\left|x_{7}-x_{3}\right|-x_{9}\right) \cdot r_{w}\right] / J_{w} \\
\dot{x}_{11}=x_{12} \\
\dot{x}_{12}=\left[u_{w y}-B_{w} \cdot x_{12}-k_{2}^{s y}\left(\left|x_{7}-x_{5}\right|-x_{11}\right) \cdot r_{w}\right] / J_{w}
\end{array}\right.
$$

where $m_{n}$ and $B_{n}$ are the equivalent mass and damping coefficient of the trawl net, and $k_{l}^{s}$ is the elasticity coefficient of the sweep lines. $l_{r z}$ and $l_{r y}$ are the length vectors of two sweep lines before they extend. is the gravity vector of the net underwater.

For the fishing vessel, $u_{s}, u_{w z}$, and $u_{w y}$ are the control inputs provided by the marine propulsion system and two trawl winches. $M_{s}$ is the inertial matrix of the vessel after coordinate transformation. $B_{s}$ is the damping force matrix of the vessel. $C_{s}$ is the Coriolis force matrix of the vessel. $T_{s}$ is the thrust of the vessel produced by the propeller and rudder, which is the control vector.

\section{TRAJECTORY TRACKING CONTROLLER DESIGNING}

The three-dimensional trajectory of the trawl net is the main control aim. The movements of two otter boards should also be highly regarded. Moreover, the state space model requires extra virtual control inputs. Thus, the tracking error of the trawl system can be defined as:

$$
\left\{\begin{array}{c}
z_{1}=x_{1}-x_{1 d} \\
\vdots \\
z_{12}=x_{12}-x_{12 d}
\end{array}\right.
$$

where $x_{l d}$ is the target trajectory of the trawl net, and $x_{2 d} \sim x_{12 d}$ are the virtual control inputs of all state variables.

Based on the backstepping method, the design process starts from the lower differential equation of Eq. (4), and the Lyapunov functions related to the control objectives can be built step by step.

Step 1. Construct a Lyapunov function as: 


$$
V_{1}=\frac{1}{2} z_{1}^{T} z_{1}
$$

The derivative of $V_{1}$ is:

$$
\dot{V}_{1}=z_{1}^{T} \dot{z}_{1}=z_{1}^{T}\left(x_{2 d}+z_{2}-\dot{x}_{1 d}\right)
$$

To ensure that $\dot{V}_{1}<0$, the virtual control inputs $x_{2 d}$ can be defined as $x_{2 d}=-k_{1} z_{1}+\dot{x}_{1 d}$ with the control parameter $k_{l}$ $\geq 0$ plugging the expression of $x_{2 d}$ into Eq. (7), the derivative of $V_{l}$ is:

$$
\dot{V}_{1}=-k_{1} z_{1}^{T} z_{1}+z_{1}^{T} z_{2}
$$

Step 2. Based on Eq. (6), a new Lyapunov function can be defined as:

$$
V_{2}=V_{1}+\frac{1}{2} z_{2}^{T} z_{2}
$$

The derivative of $V_{2}$ is:

$$
\dot{V}_{2}=\dot{V}_{1}+z_{2}^{T}\left[\left(-\hat{B}_{o} x_{2}^{2}-W_{n}+k_{1}^{s}\left(x_{3}-x_{1}-l_{r z}\right)+k_{1}^{s}\left(x_{5}-x_{1}-l_{r y}\right)\right) / m_{o}-\dot{x}_{2 d}\right]
$$

In Eq. (10) the high-order state variable $x_{5}$ appears, which means that the backstepping method cannot be used directly. Therefore, an entire variable $x_{s o}$, which is defined as $x_{s o}=x_{3}+x_{5}$, is introduced. To ensure that $\dot{V}_{2}<0$, the new virtual control inputs can be embodied as:

$$
x_{\text {sod }}=\left(m_{n} \dot{x}_{2 d}+\hat{B}_{n} x_{2}^{2}+W_{n}+2 k_{1}^{s} x_{1}-m_{n} z_{1}+k_{1}^{s}\left(l_{r z}+l_{r y}\right)-m_{n} k_{2} z_{2}\right) / k_{1}^{s}
$$

where the control parameter $k_{2} \geq 0$.

Step 3: Construct a new Lyapunov function as:

$$
V_{3}=V_{2}+\frac{1}{2} z_{3}^{T} z_{3}+\frac{1}{2} z_{5}^{T} z_{5}
$$

The derivative of $V_{3}$ is:

$$
\dot{V}_{3}=\dot{V}_{2}+z_{3}^{T}\left(z_{4}+x_{4 d}-\dot{x}_{3 d}\right)+z_{5}^{T}\left(x_{6 d}+z_{6}-\dot{x}_{5 d}\right)
$$

To ensure that $\dot{V}_{3}<0$, the virtual states $x_{4 d}$ and $x_{6 d}$ are $x_{4 d}=\dot{x}_{3 d}-k_{3} z_{3}-k_{1}^{S} m_{n} / z_{2}$ and $x_{6 d}=\dot{x}_{5 d}-k_{5} z_{5}-k_{1}^{s} z_{2} / m_{n}$. The control parameters $k_{3}$ and $k_{5}$ are positive constants. Plugging the expressions of $x_{4 d}$ and $x_{6 d}$ into Eq. (13), the derivative of $V_{3}$ is:

$$
\dot{V}_{3}=-k_{1} z_{1}^{T} z_{1}-k_{2} z_{2}^{T} z_{2}-k_{3} z_{3}^{T} z_{3}-k_{5} z_{5}^{T} z_{5}+z_{2}^{T} \frac{\omega_{n}}{m_{n}}+z_{3}^{T} z_{4}+z_{5}^{T} z_{6}
$$

Step 4: Based on Eq. (12), a new Lyapunov function can be defined as:

$$
V_{4}=V_{3}+\frac{1}{2} z_{4}^{T} z_{4}+\frac{1}{2} z_{6}^{T} z_{6}
$$

The derivative of $V_{4}$ is: 


$$
\begin{aligned}
\dot{V}_{4}=\dot{V}_{3} & +z_{4}^{T}\left[\left(-\hat{B}_{o} x_{4}^{2}-W_{o}-k_{1}^{s}\left(x_{3}-x_{1}-l_{r z}\right)+k_{2}^{s z}\left(x_{7}-x_{3}-x_{9}\right)\right) / m_{o}\right] \\
+ & z_{6}^{T}\left[\left(-\hat{B}_{o} x_{6}^{2}-W_{o}-k_{1}^{s}\left(x_{5}-x_{1}-l_{r y}\right)+k_{2}^{s y}\left(x_{7}-x_{5}-x_{11}\right)\right) / m_{o}\right]
\end{aligned}
$$

In Eq. (16), the high-order state variables $x_{9}$ and $x_{I l}$ appeared. Similar to step 2, new entire virtual control inputs $x_{s z d}$ and $x_{\text {syd }}$ can be introduced as:

$$
\left\{\begin{array}{l}
x_{s z d}=x_{7 d}-x_{9 d} \\
x_{s y d}=x_{7 d}-x_{11 d}
\end{array}\right.
$$

Obviously, there are two expressions for one vector $x_{7 d}$ in Eq. (17). The tensions of two warps are not equal when the vessel swerves, so this condition would lead to a conflict in solving $x_{7 d}$ variable.

To solve the high-order variables $x_{7 d}, x_{9 d}$, and $x_{11 d}$, a method called "S-plane control" is applied. This method is derived from the Sigmoid function, which partly embodies the idea of fuzzy control [21 - 23].

The expression of S-plane control is:

$$
f=\frac{2}{1+\exp \left(-k_{a} e-k_{b} \dot{e}\right)}-1
$$

where $k_{a}$ and $k_{b}$ are the control parameters, and $e$ and $\dot{e}$ are the inputs the express deviation and change in deviation, respectively. $f$ is the control output.

The expression of S-plane control is similar to that of PID control, but S-control is nonlinear. It is very suitable for the movement control problems of the towing system which is nonlinear and thus has a mathematical model that is difficult to acquire precisely. The essential similarities of PD control can effectively ensure the effect of motion control.

There are coupling effects among the 3-degrees of freedom motions of the vessel. To simplify the controller design, the decoupling controller is then designed as one controller for every degree. Thus, there are 3 S-plane controls for the vessel.

Assume that the target trajectory of the vessel in the X-direction is $x_{7 d x}$, the tracking error is $e_{x}=x_{7 d x}-x_{7 x}$, and the output of each $\mathrm{S}$ controller can been expressed as:

$$
X_{7 t x}=X_{\max }\left[\frac{2}{1+\exp \left(\begin{array}{l}
-k_{a} e_{x} / e_{\max } \\
-k_{b} \dot{e}_{x} / \dot{e}_{\max }
\end{array}\right)}-1+\Delta f\right]
$$

where $X_{\max }$ is the maximum output, and $e_{\max }$ is the maximum permissible error. The change in buoyancy and current can be seen as steady interference forces over a period of time. $\Delta f$ is the adjustable variable for adapting to the interference of the environment. This fixed deviation can be eliminated by adjusting the offset of the S-plane. The expression of adaptive variable $\Delta f$ can be expressed as:

$$
\left\{\begin{array}{l}
\Delta f=S\left(\Delta e_{x}, 0\right) \\
\Delta e_{X}=\gamma\left[(1-\delta) e_{t}+\delta \alpha_{t} \beta_{t} \sum_{i=t-n}^{t} e_{i}\right.
\end{array}\right.
$$

where $S\left(\Delta e_{x}, 0\right)$ is the control function of the S-plane, $e_{t}$ is the deviation at time $t$, and $\Delta e_{x}$ is the adaptive adjustment amount of the deviation. $\gamma$ is the enabled factor. When the absolute value of deviation change is less than the threshold 
value $\dot{\mathrm{e}}_{0}$, and the deviation shows an increase in tendency, $\gamma$ is set to 1 otherwise $\gamma$ is set to $0 . \alpha_{t}$ is the fading factor at time $t$. When $\gamma$ is $0, \alpha_{t}=0.9 \alpha_{t}-1$. When $\gamma$ is $1, \alpha_{t}=1$. $\beta_{t}$ is the vanish factor at time $t$. When $\Delta e$ and $e_{t}$ are opposite in sign, $\beta_{t}=0.25 \beta_{t}-1$ otherwise $\beta_{t}=1$. $\delta$ is the low-pass filter parameters $n$ is the number of historical data.

Analogously, the expression of virtual control inputs $x_{9_{d}}$ and $x_{11 d}$ are formally consistent with Eq. (19). Hence there are 9 S-plane controllers for $x_{7 d}, x_{9 d}$ and $x_{11 d}$ altogether.

With the expressions of $x_{7 d}, x_{9 d}$ and $x_{11 d}$, the recursive process is thus able to continue. Plugging the expressions of these variables into Eq. (14), the derivative $V_{4}$ of can be obtained.

Step 5: Based on Eq. (15), a new Lyapunov function is given by:

$$
V_{5}=V_{4}+\frac{1}{2} z_{7}^{T} z_{7}+\frac{1}{2} z_{9}^{T} z_{9}+\frac{1}{2} z_{11}^{T} z_{11}
$$

The derivative of $V_{5}$ is:

$$
\dot{V}_{5}=\dot{V}_{4}+z_{7}^{T}\left(x_{8 d}+z_{8}-\dot{x}_{7 d}\right)+z_{9}^{T}\left(x_{10 d}+z_{10}-\dot{x}_{9 d}\right)+z_{11}^{T}\left(x_{12 d}+z_{12}-\dot{x}_{11 d}\right)
$$

To ensure that $\dot{V}_{5}<0$, the virtual control inputs $x_{8 d}, x_{10 d}$ and $x_{12 d}$ can been defined as: $x_{8 d}=\dot{x}_{7 d}-k_{7} z_{7}-k_{2}^{s z} z_{4} / m_{o}-k_{2}^{s y} z_{6} / m_{o}, \quad x_{10 d}=\dot{x}_{9 d}-k_{9} z_{9}+k_{2}^{s z} z_{4} / m_{o} \quad$ and $\quad x_{12 d}=\dot{x}_{11 d}-k_{11} z_{11}+k_{2}^{s y} z_{6} / m_{o}$. The control parameters $k_{7}, k_{9}$ and $k_{11}$ are positive constants.

Step 6: The last Lyapunov function is given by:

$$
V_{6}=V_{5}+\frac{1}{2} z_{8}^{T} z_{8}+\frac{1}{2} z_{10}^{T} z_{10}+\frac{1}{2} z_{12}^{T} z_{12}
$$

According to the derivative of $V_{6}$, the controlled quantities of the vessel and two winches can be defined as:

$$
\left\{\begin{array}{l}
u_{s}=M_{s} \dot{x}_{8 d}+B_{s} x_{8}+C_{s} x_{8}-k_{2}^{s z}\left(\left|x_{7}-x_{3}\right|-x_{9}\right)-k_{2}^{s y}\left(x_{7}-x_{5}-x_{11}\right)-k_{8} z_{8}-M_{s} z_{7} \\
u_{w z}=J_{w} \dot{x}_{10 d}+B_{w} x_{10}+k_{2}^{s z}\left(\left|x_{7}-x_{3}\right|-x_{9}\right) r_{w}-k_{10} z_{10}-J_{w} z_{9} \\
u_{w y}=J_{w} \dot{x}_{12 d}+B_{w} x_{12}+k_{2}^{s y}\left(\left|x_{7}-x_{5}\right|-x_{11}\right) r_{w}-k_{12} z_{12}-J_{w} z_{11}
\end{array}\right.
$$

where the control parameters $k_{8} \geq 0, k_{10} \geq 0$ and $k_{12} \geq 0$. With this, the three-dimensional trajectory tracking controller of the trawl system has been acquired.

\section{STABILITY ANALYSIS}

For the proposed $\mathrm{S}$ controller, it is assumed that the real trace is equal to the desired trajectory over a long time. At this moment, the desired output of controller is $f_{0}$.

Define that $s=-k_{l} e+k_{2} \dot{e}$. A Lyapunov function can then be expressed as:

$$
V(s)=s^{2} / 2
$$

The derivative of $V(s)$ is $\dot{V}(s)=s \dot{s}$.

When $f>f_{0}$, there is $\dot{s}>0$. When $f<f_{0}$, there is $\dot{s}<0$. Therefore the following equality is established:

$$
\operatorname{sgn}(\dot{s})=\operatorname{sgn}\left(f-f_{0}\right)
$$

The sign function of $\dot{V}$ is:

$$
\operatorname{sgn} \dot{V})=\operatorname{sgn}\left[s\left(f-f_{0}\right)\right]=\operatorname{sgn}\left[s\left(\int k \dot{f} d t-f_{0}\right)\right]
$$

Because $s \dot{f}<0$, over enough time or a sufficiently large value, the following inequality is established: 


$$
\operatorname{sgn}\left[s\left(\int k \dot{f} d t-f_{0}\right)\right]=\operatorname{sgn}(s \dot{f})<0
$$

Therefore $V(s)$ is asymptotically stable.

To ensure the stability of the trawl system with a bounded input signal under the external environment disturbance, the disturbances are added to the trawl net and otter boards, which are $\omega(n), \omega(o z)$ and $\omega(o y)$.

Based on Eq. (23), and considering that the nonzero often bounded disturbances exist, a Lyapunov function can be defined as:

$$
V_{7}=V_{6}+z_{2}^{T} \frac{\omega_{n}}{m_{n}}+z_{4}^{T} \frac{\omega_{o z}}{m_{o}}+z_{6}^{T} \frac{\omega_{o y}}{m_{o}}
$$

According to the inequality $z_{2}^{T} \omega_{n} / m_{n} \leq\left(a z_{2}^{2}+\left\|\omega_{n}\right\|^{2} /(4 a)\right) / m_{n}$, the derivative $V_{7}$ of satisfies the following inequality:

$$
\begin{aligned}
\dot{V}_{6} \leq & -k_{1} z_{1}^{T} z_{1}-\left(k_{2}-\frac{a}{m_{n}}\right) z_{2}^{T} z_{2}-k_{3} z_{3}^{T} z_{3}-\left(k_{4}-\frac{b}{m_{n}}\right) z_{4}^{T} z_{4} \\
& -k_{5} z_{5}^{T} z_{5}-\left(k_{6}-\frac{c}{m_{n}}\right) z_{6}^{T} z_{6}-k_{7} z_{7}^{T} z_{7}-k_{8} z_{8}^{T} z_{8}-k_{9} z_{9}^{T} z_{9} \\
& -k_{10} z_{10}^{T} z_{10}-k_{11} z_{11}^{T} z_{11}-k_{12} z_{12}^{T} z_{12}+\frac{\left\|\omega_{n}\right\|^{2}}{4 a m_{n}}+\frac{\left\|\omega_{o}\right\|^{2}}{4 b m_{o}}+\frac{\left\|\omega_{o}\right\|^{2}}{c b m_{o}}
\end{aligned}
$$

The definite integrals of Eq. (30) can be expressed as:

$$
V\left(x\left(t_{f}\right)\right)-V(x(0)) \leq \rho^{2} \int_{0}^{t_{f}} \omega^{T}(t) \omega(t) d t-\int_{0}^{t_{f}} z^{T}(t) z(t) d t
$$

Thus, Eq. (31) proves that in the presence of interference with an unknown upper limit, the system is asymptotically stable and the control outputs would converge to the origin of a small domain in the stable area.

\section{SIMULATION EXPERIMENT}

To simulate the three-dimensional motion tracking of a semi-pelagic trawl net in a complex seabed terrain, the target trajectory of the trawl net is defined as a nonlinear time-varying function. The semi-pelagic trawl system is assumed to move in uniform flow. The masses of four mass points are defined as $m_{s}=5718000 \mathrm{~kg}, m_{o}=5200 \mathrm{~kg}, m_{n}=$ $47916 \mathrm{~kg}$. The lengths of the warp and sweep lines are $l_{w}=3000 \mathrm{~m}$ and $l_{r}=206 \mathrm{~m}$. The expression of $x_{I d}$ is given by:

$$
\left\{\begin{array}{l}
x_{1 d}(x)=R \sin \left(w_{1} t\right) \\
x_{1 d}(y)=\sum_{n=1}^{N}[\varphi(t)+F(t)] \\
x_{1 d}(z)=40 \sin \left(w_{2} t\right)-180
\end{array}\right.
$$

where $\varphi(t)=R \cos \left[w_{1}(t-1000 N)\right](-1)^{N+1}+R$,

$$
\begin{aligned}
& F(t)=\left\{\begin{array}{cc}
0 & 0 \leq t \leq 1000 \\
R \cos \left(1000 \mathrm{w}_{1}\right)(-1)^{N} & t>1000
\end{array} \quad N=\operatorname{int}\left(\frac{t}{1000}-1\right), \quad w_{1}=0.001 \mathrm{rad} / \mathrm{s},\right. \\
& w_{2}=0.002 \mathrm{rad} / \mathrm{s}, R=3000 \mathrm{~m}, t \in[0,5000](\mathrm{s}) .
\end{aligned}
$$

When the time $\mathrm{t}=1000 \mathrm{~s}$, a disturbing force $\omega_{0}$ acts on the otter board on the left side, which is removed at time $\mathrm{t}=2000 \mathrm{~s}$. At time $\mathrm{t}=1500 \mathrm{~s}, \omega_{0}$ acts on the trawl net, and it is removed at time $\mathrm{t}=2500 \mathrm{~s} . \omega_{0}$ is defined as $6 \times 10^{5}(N)$ in 
three directions.

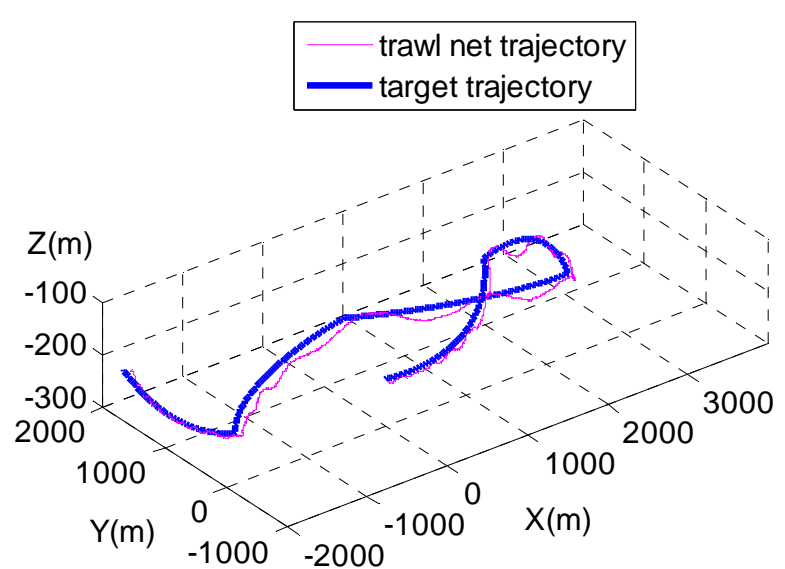

(a)

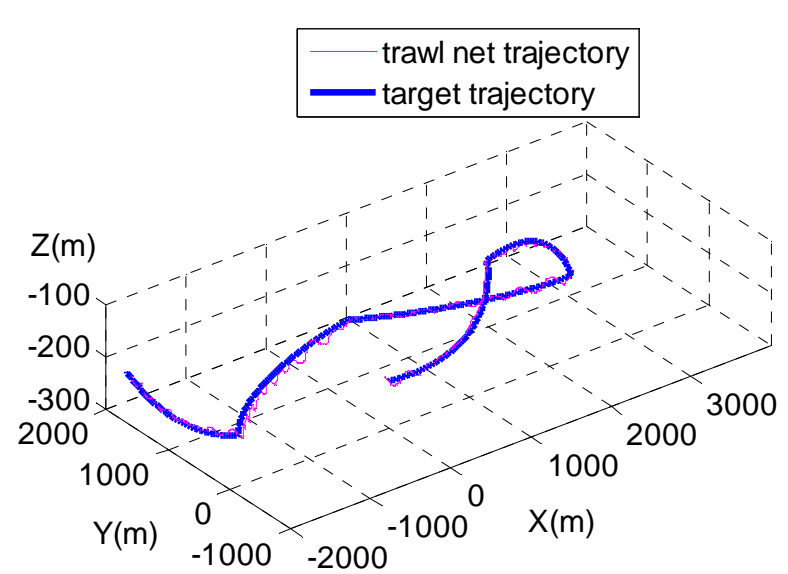

(b)

Fig. (3). The tracking trajectories of the trawl system without disturbing forces.

As shown in Eq. (28), the target trajectory is rather complex. For comparison, the conventional linear controller is also given. The condition that the semi-pelagic trawl moves without disturbing forces is simulated first. The simulation result of the linear controller is shown in Fig. (3a), and the result of the improved backstepping controller is shown in Fig. (3b). Compared with these two results, it can be seen that the track curve of the conventional linear controller has obvious deviation. In contrast, the deviation has been effectively reduced under the action of the proposed control method.

Fig. (4) shows the trajectories of the trawl net under the disturbing forces. Similar to Fig. (3), Fig. (4a) shows the trajectory of the linear controller and Fig. (4b) shows the trajectory of the proposed controller. Apparently, the path deviation in the Z-direction is larger than in other directions during the action period of the disturbing forces. The trajectory tracking errors of the proposed controller are much lower than in the linear controller. When the disturbing forces disappear, the proposed controller can eliminate the deviation rapidly and stably, whereas the trajectory of the linear controller still has oscillation for a long time.

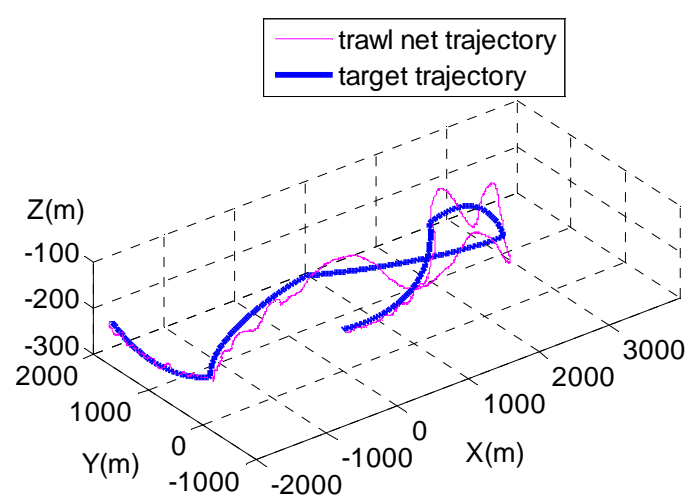

(a)

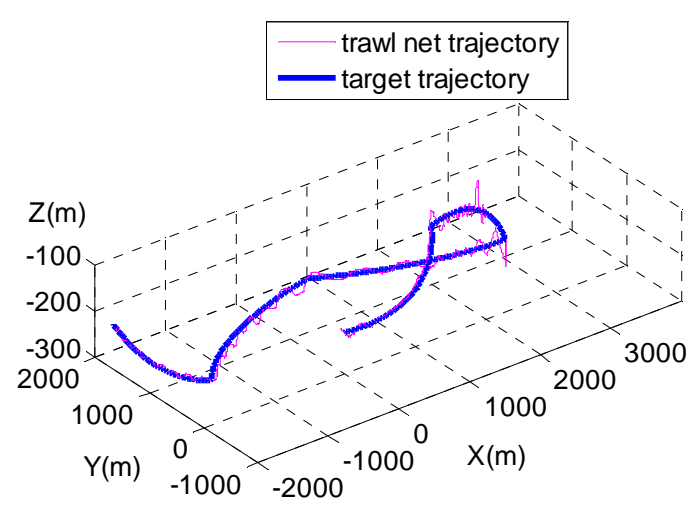

(b)

Fig. (4). The tracking trajectories of the trawl system with disturbing forces.

For further quantitative analysis, the real-time relative error comparison of these two controllers has been provided. As shown in Fig. (5), in the X-direction, the tracking errors of two controllers are nearly the same. In the Y-direction, the two tracking relative error curves both have mutations at time $\mathrm{t}=2000 \mathrm{~s}$ and $\mathrm{t}=4000 \mathrm{~s}$. This may be caused by the reverse direction of velocity. However, for most of the time, the proposed controller has much lower tracking error. In 
the Z-direction, the tracking error of the linear controller is much larger, sometimes even higher than 0.1. The proposed controller can still maintain the tracking error at a low level.
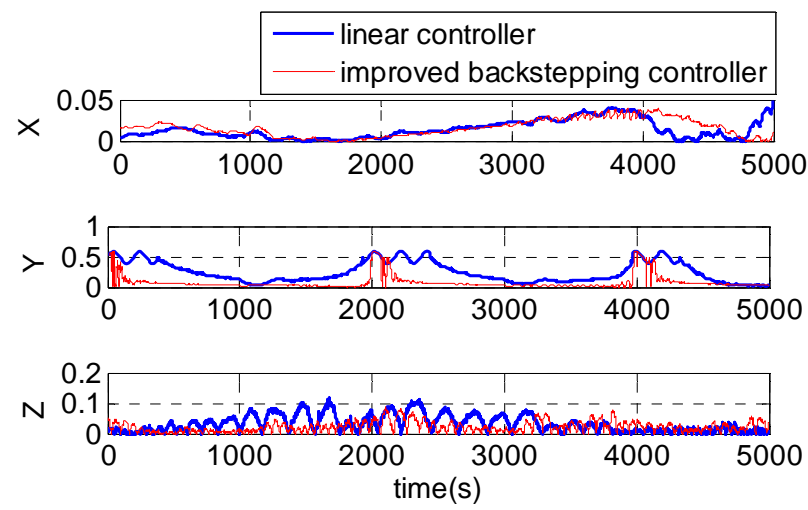

Fig. (5). Ratio of the tracking relative error without disturbing forces.

Fig. (6) shows the real-time relative error comparison in the presence of a disturbance force. It can be seen that when the disturbing force exists, the tracking errors in the Y-direction have changed little. In the Z-direction, the maximum relative error increases to more than 0.3. After the disturbing force disappears, the improved backstepping controller can rapidly reduce the error to the previous level.
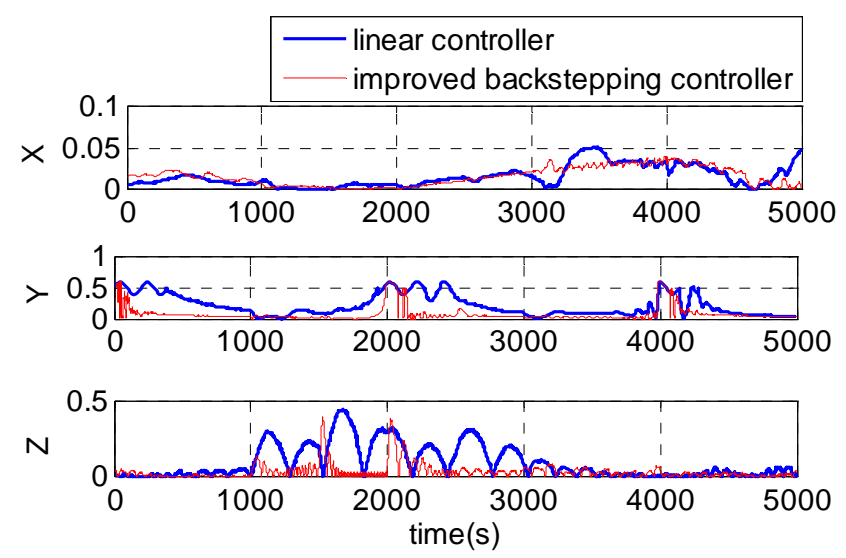

Fig. (6). Ratio of the tracking relative error with disturbing forces.

\section{CONCLUSION}

According to the actual working status of a semi-pelagic trawl system, a new simplified model is presented in this paper. By using the backstepping method and S-plane control in combination, a compound nonlinear controller considering decoupling of the high-order state variables in the process of recursion is presented. The robustness and stability of this system under constant external disturbances have been proved based on Lyapunov stability theory. Finally, the simulation results show the superiority and effectiveness of the proposed control approach. When a constant disturbance force acts on the system, the depth location of the trawl net changes more than the horizontal position. In future work, a model experimental demonstration will be presented to verify the effectiveness of the proposed control scheme in practical applications, and further modification of the control model will be considered to improve the model accuracy in a complex flow field.

\section{CONFLICT OF INTEREST}

The authors confirm that this article content has no conflict of interest. 


\section{ACKNOWLEDGEMENTS}

The authors would like to acknowledge Senior Engineer C. G. Liu of the China Ship Development and Design Center and Assistant Engineer J. Y. Cui of the Wuhan Ship Development Design Institute for their assistance.

\section{REFERENCES}

[1] D. Brewer, S. Eayrs, R. Mounsey, and Y.G. Wang, "Assessment of an environmentally friendly, semi-pelagic fish trawl", Fish. Res., vol. 26, pp. 225-237, 1996. [http://dx.doi.org/10.1016/0165-7836(95)00433-5]

[2] K.J. Reite, and A.J. Sorensen, "Mathematical modeling of the hydrodynamic forces on a trawl door", IEEE J. Oceanic Eng., vol. 31, no. 2, pp. 432-453, 2006. [http://dx.doi.org/10.1109/JOE.2006.875098]

[3] N. Umeda, "Optimal Control of a Midwater Trawl System", In: Proceedings of the Fourth Pacific Congress on Marine Science and Technology, Tokyo, 1990, pp. 507-514.

[4] C.W. Lee, and J.H. Lee, "Modeling of a midwater trawl system with respect to the vertical movements", Fish. Sci., vol. 66, no. 5, pp. 851-857, 2000 .

[http://dx.doi.org/10.1046/j.1444-2906.2000.00138.x]

[5] C. W. Lee, C. L. Zhang, and H. O. Shin, "Simplified trawl system modeling and design of a depth control system using fuzzy logic", Fish. Res, vol. 53, no. 1, pp. 83-94, 2001. [http://dx.doi.org/10.1016/S0165-7836(00)00264-2]

[6] V. Johansen, O. Egeland, and A.J. Sorensen, "Modelling and control of a trawl system in the transversal direction", Cont. App. Mar. Sys., vol. 2001, pp. 243-248, 2002.

[7] K.J. Reite, and A.J. Sorensen, "Hydrodynamic properties important for control of trawl doors", In: IFAC Conference on Control Applications in Marine Systems (CAMS 2004). Italy, pp. 143-148, 2005.

[8] K.J. Reite, Modeling and Control of Trawl Systems., Norwegian University of Science and Technology: Norway, 2006 , pp. 153-192.

[9] H. Zhou, Y.L. Chen, and H.Y. Yang, "Robust optimal output tracking control of a midwater trawl system based on T-S fuzzy nonlinear model", China Ocean Eng., vol. 27, no. 1, pp. 1-16, 2013. [http://dx.doi.org/10.1007/s13344-013-0001-4]

[10] Y.L. Chen, H. Zhou, Y.G. Zhao, and J.Y. Hou, "Fuzzy robust path tracking strategy of an active pelagic trawl system with coordinated ship and winch regulation", J. Central South Univ., vol. 21, no. 1, pp. 167-179, 2014. [http://dx.doi.org/10.1007/s11771-014-1928-1]

[11] J.C. Yu, Q. Li, and A.Q. Zhang, "Neural network adaptive control for underwater vehicles", Cont. Theory App., vol. 25, no. 1, pp. 9-13, 2008.

[12] H.M. Jia, L.J. Zhang, and X.Q. Cheng, "Three dimensional path following control for an underactuated UUV based on nonlinear iterative sliding mode", Acta Autom. Sin., vol. 38, no. 2, pp. 308-314, 2012. [http://dx.doi.org/10.3724/SP.J.1004.2012.00308]

[13] K.D. Do, J. Pan, and Z. Jiang, "Robust and adaptive path following for underactuated autonomous underwater vehicles", Ocean Eng., vol. 31, no. 16, pp. 1967-1997, 2004.

[http://dx.doi.org/10.1016/j.oceaneng.2004.04.006]

[14] E.R. Jon, J.S. Asgeir, and Y.P. Kristin, "Model-based output feedback control of slender-body underactuated AUVs: theory and experiments", IEEE T. Cont. Syst. Tech., vol. 16, no. 5, pp. 930-946, 2008. [http://dx.doi.org/10.1109/TCST.2007.916347]

[15] D.Q. Zhu, and R.R. Yang, "Backstepping tracking control of autonomous underwater vehicles with bio-inspired neur dynamics model", Cont. Syst. Tech., vol. 29, no. 10, pp. 1309-1316, 2012.

[16] B. Sun, D.Q. Zhu, and Z.G. Deng, "Bio-inspired discrete trajectory-tracking control for open-frame underwater vehicles", Cont. Sys. Tech., vol. 30, no. 4, pp. 454-462, 2013.

[17] J. Xu, M. Wang, and L. Qiao, "Backstepping-based controller for three-dimensional trajectory tracking of under-actuated unmanned underwater vehicles", Cont. Syst. Tech., vol. 31, no. 11, pp. 1589-1596, 2014.

[18] J. Xu, M. Wang, and L. Qiao, "Dynamical sliding mode control for the trajectory tracking of underactuated unmanned underwater vehicles", Ocean Eng., vol. 105, pp. 54-63, 2015.

[http://dx.doi.org/10.1016/j.oceaneng.2015.06.022]

[19] T. Fossen, Marine Control Systems: Guidance, Navigation and Control of Ships, Rigs and Underwater Vehicles., Marine Cybernetics Press, 2002.

[20] Y.L. Chen, "Research on Modeling and Control Strategies for the Trawling system", M.S. thesis, Zhejiang University, Zhejiang, 2013.

[21] J.C. Liu, H.N. Yu, and Y.R. Xu, "Improved S plane control algorithm for underwater vehicles", J. Harbin Eng. Univ., vol. 23, no. 1, pp. 33-36, 2002. 
[22] Y.M. Li, Y.J. Pang, and L. Wan, "Adaptive S plane control for autonomous underwater vehicle", J. Shang Hai Jiao Tong Univ., vol. 46, no. 2, pp. 195-200, 2012.

[23] G.C. Zhang, L. Zhang, L. Wan, and J.Q. Li, "Predictive S control of AUV based on model of support vector m achine", Adv. Mat. Res., vol. 340, pp. 421-428, 2012.

[http://dx.doi.org/10.4028/www.scientific.net/AMR.340.421]

(C) Yan et al.; Licensee Bentham Open

This is an open access article licensed under the terms of the Creative Commons Attribution-Non-Commercial 4.0 International Public License (CC BY-NC 4.0) (https://creativecommons.org/licenses/by-nc/4.0/legalcode), which permits unrestricted, non-commercial use, distribution and reproduction in any medium, provided the work is properly cited. 